\title{
¿CÓMO SE HA REALIZADO LA IMPLEMENTACIÓN DE LOS ACUERDOS DE BASILEA III EN LATINOAMÉRICA Y QUE EFECTOS HAN TENIDO ESTOS EN EL SECTOR FINANCIERO EN CHILE Y COLOMBIA?
}

\author{
Anne Monique Pulgarín Molano \\ Facultad de Negocios, Gestión y Sostenibilidad \\ Programa de Especialización en Gerencia de Finanzas \\ Especialista en Gerencia de Finanzas \\ monique.3093@ hotmail.com \\ Angélica Domínguez Rodríguez \\ Facultad de Negocios, Gestión y Sostenibilidad \\ Programa de Especialización en Gerencia de Finanzas \\ Especialista en Gerencia de Finanzas \\ angelicadominguezr@hotmail.com
}

\section{Resumen}

Un sector financiero estable, robusto y confiable es parte fundamental del desarrollo de una economía, por esto surge la necesidad de implementar acuerdos que regulen estandaricen y disminuyan el riego de sus actividades En este marco surgen los acuerdos de Basilea que buscan minimizar los efectos adversos que situaciones de crisis puedan generar sobre la economía en general. Este articulo pretende hacer una descripción de los acuerdos de Basilea, realizando una revisión documental sobre la aplicación a lo largo del tiempo de cada una de las versiones y sus implicaciones, se tomarán como países de referencia Chile y Colombia describiendo el efecto que han tenido en el sistema financiero, así como los retos y ventajas competitivas que se han presentado. Se explicará porque los acuerdos de Basilea traerán consigo mayor estabilidad y confianza, así como un sistema financiero más robusto y capaz de enfrentar situaciones de estrés, pero que también se debe tener en cuenta las generalidades que afecta al sistema financiero de cada economía, y debe aplicarse con la gradualidad necesaria para que el sistema bancario y financiero local pueda asumir el desafío de hacer frente a este nuevo marco regulatorio sin comprometer ni resentir su estabilidad y solvencia.

Palabras clave: Regulación, entidades financieras, Acuerdos de Basilea, solvencia, supervisión, crisis, riesgo, liquidez, capital, patrimonio técnico. 


\section{Introducción}

Diversas crisis financieras, en especial la crisis Subprime (2008) han dejado en evidencia serias fallas regulatorias y de revisión en los sistemas financieros del mundo desarrollado, junto con esto se produjo un acelerado crecimiento en las instituciones bancarias a tal punto que se convertían en agentes de alto riesgo para las economías de algunos países como Estados Unidos. Como era de esperarse, el colapso de estas organizaciones se reflejó en graves daños al sistema económico a través de la pérdida de empleos, embargos de vivienda, reducciones en el PIB y contracción del crédito, representado en una clara crisis de liquidez. Para evitar un mayor impacto de la crisis muchos gobiernos tuvieron que intervenir y salvar a grandes instituciones financieras con dinero de los contribuyentes.

Como resultado de lo anterior, el comité de Basilea para la supervisión bancaria emprendió, con el apoyo de la mayoría de bancos centrales, la tarea de generar una serie de recomendaciones que ayuden a generar un sistema financiero más estable mediante la mejora de los esquemas regulatorios y de supervisión bancaria. A su vez, una mayor estabilidad financiera ayudara a producir un crecimiento económico sólido, por ejemplo; mayores requerimientos de capital (principal pilar de Basilea), convierten a los bonos emitidos por entidades financieras privadas en productos más seguros.

Sin embargo, debe tenerse en cuenta que las regulaciones bancarias estarían ligadas no solo a reducir la posibilidad de futuras crisis sino también pueden llegar a frenar un posible crecimiento económico futuro. Esto se debe a que los préstamos bancarios y la provisión de crédito se encuentran altamente restringidas por el gran número de restricciones plasmadas en los acuerdos de Basilea, esto tiene como consecuencia colateral una ralentización del crecimiento económico. (Trujillo, 2015)

América Latina no fue ajena a esta crisis, organismos internacionales señalaban que la región no se mantendría al margen de la debacle de la economía mundial. "La idea de que estábamos fuera de la crisis era una ilusión y los hechos lo vienen demostrando cada día", resume el Secretario General Iberoamericano, Enrique Iglesias, al inaugurar el II Foro Europa-América Latina-EEUU. Por igual, la Comisión Económica para América Latina (CEPAL) (2016), en su informe Panorama de la inserción internacional de América Latina y el Caribe, señala que: "El quiebre del ciclo expansivo mundial afectará negativamente a la región.

En Colombia, la crisis financiera impactó a la economía a través de varias rutas. La desaceleración del crecimiento mundial afectó el dinamismo de las exportaciones colombianas y disminuyó los precios del carbón, el petróleo, el ferroníquel, etc. La liquidez mundial también disminuyó como resultado de la crisis, lo que afectó adversamente los flujos de inversión. La menor inversión, a su vez, llevó a una depreciación de la moneda colombiana, y también a una caída de la bolsa y a un aumento del costo de la deuda. (El Espectador, 2008) 
De acuerdo con Heresi (2011), en el caso de la economía chilena, esta sufrió el impacto de la crisis internacional de una manera más tenue pues la crisis encontró al país en una mejor posición que en episodios anteriores. Con un sistema bancario bien regulado y capitalizado, un fisco que por primera vez en su historia es acreedor neto del resto del mundo y (previo a la crisis) un significativo superávit en la cuenta corriente. Sin embargo, durante septiembre y octubre de 2008, abruptamente se revirtieron las trayectorias de los precios de las materias primas, la inflación y el tipo de cambio, mientras el superávit externo se transformó rápidamente en un déficit.

Dicho esto, surge la necesidad de identificar el efecto que tiene estas recomendaciones en el sistema financiero de los países latinoamericanos, identificando los retos que deben enfrentan estos países al aplicar las recomendaciones. Para este caso se tomarán como referencia las economías de Colombia y Chile.

Para desarrollar el tema se realizará una revisión documental de la información disponible acerca del origen y fundamentos de los acuerdos de Basilea, así como la aplicación de estos en Latinoamérica tomando como países de referencia Chile y Colombia, se realizará una comparación entre ambos países identificando los beneficios y retos que los acuerdos de Basilea III representan para sus economías.

Este articulo tiene como objetivo identificar como se ha realizado la implementación de los acuerdos de Basilea III y que efecto han tenido en el sector financiero de Colombia y Chile, para desarrollar esto se pretende realizar una descripción histórica de los diferentes acuerdos aprobados (Basilea I, II Y III), se dará especial importancia a los acuerdos de Basilea III que se encuentran vigentes en la actualidad, por esto se explicará al detalle los pilares que lo conforman. Seguido se describirá como se están aplicando los acuerdos y cuál ha sido su efecto en el sector financiero, identificando los retos y oportunidades que estos acuerdos generaron a las economías de los países anteriormente señalados.

\section{Origen del Comité de Basilea}

El Comité de Basilea tuvo su origen a raíz de la crisis financiera originada por el cierre del Bankhaus Hersttat en Alemania en 1974. Dicho banco fue cerrado por el banco central alemán, el Bundesbank, por sus importantes pérdidas derivadas de sus operaciones en moneda extranjera. Como consecuencia del cierre de sus actividades un número considerable de obligaciones bancarias internacionales quedaron sin realizarse ya que el Chase Manhattan, banco corresponsal del Herstatt en EEUU, se rehusó a cumplir con órdenes de pago y cheques girados contra la cuenta de dicho banco. Estas difíciles circunstancias por poco condujeron al colapso del sistema de pagos estadounidense y del sistema financiero internacional (Sotelo, 2008).

Dicha situación produjo que los dirigentes de los principales bancos centrales del G10, establecen el primer comité de Basilea, comité encargado de la supervisión bancaria y cuyo fin era desarrollar principios y reglas apropiadas sobre prácticas de regulación y supervisión 
de los mercados bancarios internacionales que eviten la ocurrencia de crisis similares en el futuro (Sotelo, 2008)

El comité no posee ninguna autoridad supervisión formal y sus conclusiones no tienen fuerza legal, el Comité formula amplios estándares y pautas de supervisión y emite declaraciones de mejores prácticas con la expectativa de que las autoridades de supervisión individuales tomen medidas para ponerlas en ejecución con los arreglos que mejor se adapten a cada sistema nacional. (Economipedia)

\section{Acuerdos de Basilea}

Los acuerdos de Basilea han evolucionado en función de los cambios en el sistema financiero, siempre con el fin de reducir al máximo el endeudamiento de las entidades financieras y garantizar la capacidad de respuesta ante el riesgo operacional, de crédito y de mercado.

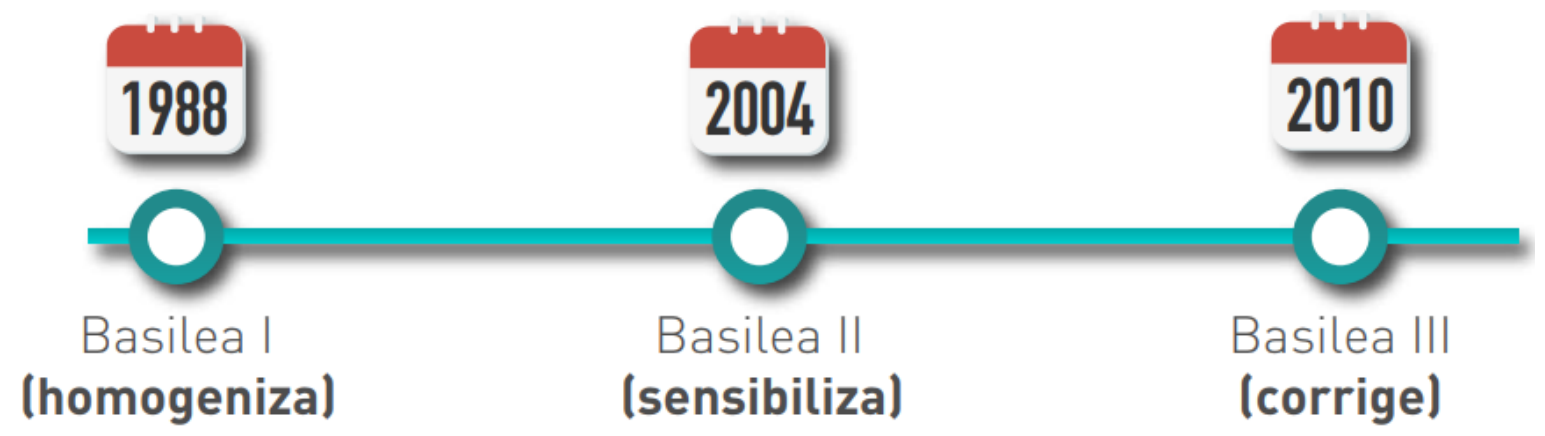

Imagen 1 Acuerdo de Basilea

Fuente: Superintendencia de bancos e instituciones financieras de Chile.

\section{Basilea I}

Este acuerdo se firmó en 1988, estableció unos principios básicos en los que debía fundamentarse la actividad bancaria como el capital regulatorio, requisito de permanencia, capacidad de absorción de pérdidas y de protección ante quiebra. (Powerdata) Recomendaciones: (Sotelsek Salem \& Pavón Cuéllar, 2012) (Jiménez, Held, \& Romero, 2008)

- Fijar un límite a la emisión de créditos que una entidad concede en función del capital que tiene. Se estableció que el capital mínimo debía ser, al menos, un $8 \%$ de los activos ponderados por su riesgo (Riesgo de crédito).

- Limitar el apalancamiento o el efecto multiplicativo de la inversión de las entidades financieras en 12,5 veces el valor de los recursos propios en sus hojas de balance, esto tenía vital importancia ya que en ese momento la mayoría de las empresas tenían un alto grado de apalancamiento al captar recursos y conceder cerditos sin tener en cuenta los estándares mínimos de riesgo de crédito. 
- Se estableció la definición de capital regulatorio dividido en dos categorías llamadas Tier I y Tier II, en función de sí cumplían ciertos requisitos en cuanto a la capacidad de absorber pérdidas, así como su permanencia y protección ante una quiebra. (Economipedia)

Tier I: Se define como el "capital básico" y está compuesto por el capital accionario y reservas declaradas. Este capital representa el aporte patrimonial de los accionistas y permite cubrir pérdidas inesperadas en forma inmediata y sin restricciones.

Tier II: Se define como el "capital complementario". Los componentes del capital complementario son reservas no declaradas, reservas de revalorización de activos, provisiones generales por riesgo de crédito, deuda subordinada y otros instrumentos híbridos de deuda/capital.

Dado que el acuerdo contenía ciertas limitaciones en su definición, en junio de 2004 fue sustituido por el llamado acuerdo Basilea II.

\section{Basilea II}

Uno de los cambios principales en estos acuerdos fue tener en cuenta la capacidad de una persona o una empresa para devolver los préstamos concedidos en un tiempo menor al establecido y prestar especial atención al tiempo de recuperación del crédito, debido a esto cobro importancia el riesgo de crédito.

Dicho esto, se decide crear tres pilares, lo cuales fundamentan los acuerdos de Basilea II

Pilar I "Disponer de unos requisitos mínimos de capital". (Banco de Pagos Internacionales, 2004)

- Para esto se tiene en cuenta el riesgo de crédito, pero a diferencia de Basilea I se tiene en cuenta la calidad de los prestatarios utilizando ratings o calificaciones crediticias

- Se exige que el coeficiente de recursos propios de los bancos sea superior al $8 \%$ y añade requisitos de capital por el riesgo operacional, en esta evaluación se tiene en cuenta la probabilidad de incumplimiento del crédito, la pérdida en el momento en el que se produce el incumplimiento

En este pilar, el objetivo es cuantificar el riesgo de crédito, de mercado y operacional. De esta forma, se utilizan diferentes modelos propios de evaluación de riesgos existiendo incentivos a la mejora de estos modelos.

Pilar II:” Supervisar la gestión de los fondos propios" 
Este pilar recomienda que las entidades encargadas de realizar la supervisión en cada país tengan especial atención a que las entidades mantengan un capital suficiente en función de los riesgos incurridos. A su vez, deben estudiar el nivel de solvencia de la entidad mediante la validación de modelos estadísticos, [por otra parte las entidades financieras están en la obligación de almacenar datos de información crediticia durante períodos largos de 5 a 7 años, garantizar su adecuada auditoria y a superar pruebas de stress test. (Superintendecia de Banca, Seguros y AFP )

Pilar III:" Disciplina de mercado"

En este pilar se pretende que la entidad proporcione información crediticia y del nivel de riesgo de los mercados financieros de forma transparente y con una correcta descripción de la gestión de riesgos, aspectos técnicos de cálculo de capital, descripción de la gestión de capital y requerimientos de capital por cada tipo de riesgo. (Superintendecia de Banca, Seguros y AFP).

\section{Diferencias entre Basilea I y Basilea II}

A continuación, se presenta la comparación entre Basilea I y Basilea II con base en cinco criterios:

\begin{tabular}{|c|c|c|}
\hline Criterio & Basilea I & Basilea II \\
\hline Medición Riesgo de crédito & $\begin{array}{l}\text { Medición a partir de la aplicación de } \\
\text { ponderaciones dadas por el regulador }\end{array}$ & $\begin{array}{l}\text { Medición a partir de aplicación de } \\
\text { ponderaciones externas (calificadoras) o por } \\
\text { métodos internos. }\end{array}$ \\
\hline Calculo Riesgo de crédito & Por medio del enfoque estandarizado & $\begin{array}{c}\text { Cálculo del Riesgo Crediticio mediante } 3 \\
\text { métodos: } \\
\text { 1. Estandarizado } \\
\text { 2. IRB (fundacional) } \\
\text { 3. RB (avanzado) }\end{array}$ \\
\hline Riesgo de Mercado & $\begin{array}{c}\text { Incorpora la medición del Riesgo de } \\
\text { Mercado desde } 1996\end{array}$ & Permanece Igual \\
\hline OCDE & $\begin{array}{l}\text { Países de la OECD reciben un trato } \\
\text { preferencial }\end{array}$ & $\begin{array}{l}\text { No existe trato diferenciado para los países } \\
\text { miembros de la } O E C D\end{array}$ \\
\hline Requerimiento de capital & $\begin{array}{l}\text { No incluye posibilidad de } \\
\text { requerimiento adicional por otros } \\
\text { riesgos }\end{array}$ & $\begin{array}{l}\text { El Pilar } 2 \text { da la posibilidad al ente supervisor } \\
\text { de requerir mayor capital por otros riesgos }\end{array}$ \\
\hline
\end{tabular}

Tabla 1 Diferencias entre Basilea I y Basilea II

Fuente: Elaboración propia

\section{Basilea III}

Como respuesta a la reciente crisis financiera el comité de Basilea elaboro un nuevo marco de supervisión, tomando en consideración las falencias detectadas en Basilea II, así como los riesgos no contemplados en los acuerdos anteriores. La nueva regulación aumenta los requerimientos de capital, y tiene un marcado énfasis en la adecuada gestión de la liquidez 
de las instituciones financieras, así como en el riesgo sistémico que las instituciones de gran tamaño representan para el sistema financiero internacional.

\section{Basilea VI}

Mantiene los tres pilares del acuerdo anterior pero ahora con mayores requisitos en cuento a la calidad y al nivel de capital. Además, incorpora requerimientos de capital por riesgo sistémico, mediciones de liquidas de corto y largo plazo. Buscando así mejorar la capacidad de afrontar imprevistos o perturbaciones que sean ocasionadas por agentes macroeconómicos como una tensión financiera o económica, lo anterior reforzando la gestión de riesgos, el buen gobierno, la trasparencia y la divulgación de información. (Trujillo, 2015)

De acuerdo a lo anterior, se establecieron nuevas recomendaciones tales como:

- Endurecimiento de los criterio y aumento de la calidad del volumen de capital para asegurar su mayor capacidad para absorber perdidas.

- Modificaciones de los criterios de cálculo de los riesgos para disminuir el nivel de exposición real.

- Constitución de colchones de capital durante los bueno tiempos que permitan hacer frente al cambio de ciclo económico.

- Introducción de una nueva ratio de apalancamiento como medida complementaria a la ratio de solvencia.

\section{Reformas de Basilea III}

Según la (BIS, s.f.), a continuación, se detallan las reformas que se aplicaron a Basilea III:

\section{$\underline{\text { Primer Pilar }}$}

Capital: Los bancos están obligados a mantener más capital y de mayor calidad, lo anterior con el fin que estos tengan la capacidad de absorber pérdidas en el momento que el mismo se vuelva inviable mediante la conversión en acciones ordinarias de los instrumentos de capital; así se reducirá el riesgo incrementando la contribución del sector privado.

El capital de Nivel 1 (acciones ordinarias, beneficios no distribuidos y otras reservas) pasan al $6 \%$ y por lo menos tres cuartas partes deben ser de calidad máxima.

Cobertura del Riesgo: Mejorar la sensibilidad al riesgo y la comparabilidad de cada uno de los riesgos mediante requisitos más estrictos que permitan medir la exposición, sustentando y garantizando así un mejor sistema financiero.

Coeficiente de Apalancamiento: Contribuye a contener la acumulación de apalancamiento en el conjunto del sistema a través del respaldo del requerimiento de capital basado en el riesgo.

\section{$\underline{\text { Segundo Pilar }}$}

Gestión y Supervisión del Riesgo: Las buenas prácticas son la base fundamental de este pilar, que se centra en la gobernanza y gestión de riesgos realizando prácticas que sean de 
retribución sólida para el banco. A lo anterior, se le incluye la gestión del riesgo de tasas de interés en la cartera de inversión con requisitos de divulgación mejorados que permitan ser más estrictos, logrando así identificar bancos atípicos.

\section{$\underline{\text { Tercer Pilar }}$}

Disciplina de Mercado: La divulgación más detallada de los componentes del capital regulador y su conciliación con las cuentas declaradas será una exigencia, al igual la explicación completa sobre cómo calcula el banco sus coeficientes de capital regulador.

\section{Acuerdos de Basilea en Latinoamérica}

De acuerdo con los cálculos del FMI para 2011, la solvencia del sistema financiero en países como Colombia, Chile, Brasil, México y Perú, cumplían con los requisitos planteados en Basilea III, teniendo en cuenta que la calidad del capital, en términos generales, era buena, y el margen regulatorio promedio en solvencia era superior al $10 \%$. Sin embargo, existían desafíos en cuanto a la definición homogénea (entre países) de las clases de capital, las normas de contabilidad, la calidad de la supervisión y las técnicas de manejo del riesgo, elementos que podrían afectar directamente a los bancos con presencia en Latinoamérica que operan a escala internacional. (Asociación Nacional de Instituciones Financieras, ANIF, 2012).

Colombia y Ecuador tendrían que adelantar importantes ajustes para acoplarse a Basilea III, pues los requerimientos de limpieza del capital podrían implicar disminuciones en la relación de solvencia en el orden de 2.2 - 2.5 puntos porcentuales, pero aun así estarían cumpliendo con el nivel mínimo de solvencia. (Asociación Nacional de Instituciones Financieras, ANIF, 2012).

En los casos de Perú y Bolivia los impactos serían mucho menores, por ejemplo, según Rojas y Suarez (2012), Perú se vería afectado principalmente en sus instrumentos de deuda soberana y en la eliminación de capital Tier 3, donde la relación de solvencia se reduciría marginalmente, manteniéndose en el 13.7\%. En el caso de Bolivia, la afectación vendría por vía de las donaciones, pero su relación de solvencia permanecería en el $12 \%$.

Otros países de Latinoamérica también tendrían reducciones simulares en sus indicadores de solvencia por cuenta de la implementación de Basilea III, Por ejemplo, México el indicador de solvencia bajaría de $17.3 \%$ al $15.3 \%$, principalmente por la eliminación de las obligaciones subordinadas (Asobancaria, Basilea III y los impactos preliminares en México. , 2010).

En el caso de Brasil, su gremio bancario (Mandi, 2012) ha estimado que cerca del $40 \%$ del patrimonio de referencia (equivalente al capital total requerido) está por fuera de los patrones de calidad de Basilea III. Una depuración de capital en Brasil conduciría a reducir su índice de solvencia. 
El siguiente gráfico muestra en qué estado se encuentra aplicados los acuerdos de Basilea, en países como Canadá, Perú, México, España y Estados Unidos, se encuentran en vigencia los acuerdos de Basilea III. Chile, Colombia, Puerto Rico, y Argentina se encuentran en Basilea III, esta es una transición entre los acuerdos de Basilea II y Basilea III. De resto de países de Latinoamérica y el Caribe se encuentran en la aplicación de Basilea II a excepción de países como Venezuela, Haití y Honduras que se encuentran en Basilea I.

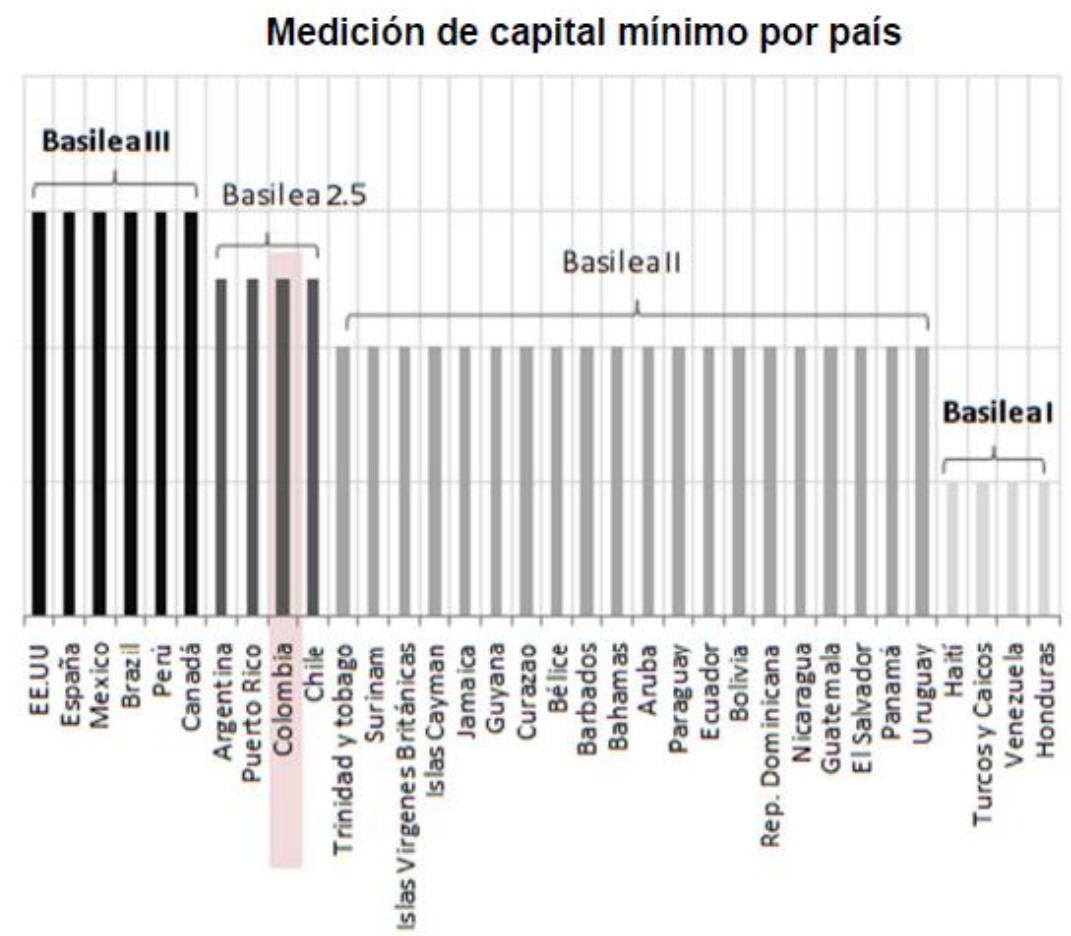

Grafico 1 Medición de capital mínimo por país

Fuente: Superintendencia Financiera de Colombia

\section{Acuerdos de Basilea en Colombia}

\section{Contextualización del sistema financiero colombiano}

El sistema financiero colombiano está conformado por los establecimientos de crédito, las entidades de servicios financieros y otras entidades financieras, las cuales, en su mayoría, se ha agrupado mediante la figura de conglomerados financieros, haciendo presencia tanto en el ámbito interno como externo. Luego de la crisis financiera de finales de la década de los noventa, este sector se ha venido fortaleciendo gracias, entre otras cosas, a la regulación del gobierno nacional y de la Superintendencia Financiera de Colombia, lo que se ha reflejado en bueno indicadores de rentabilidad, riesgo y solvencia. (Uribe, 2013).

En la última década el sector financiero colombiano presentó un crecimiento importante en término de activos, como se evidencia en la siguiente gráfica, ha tenido de más del $200 \%$ en sus activos, pasando de 365 Billones en el año 2006 a 1.358 billones en el año 2016. (SFC S. F., 2016) 


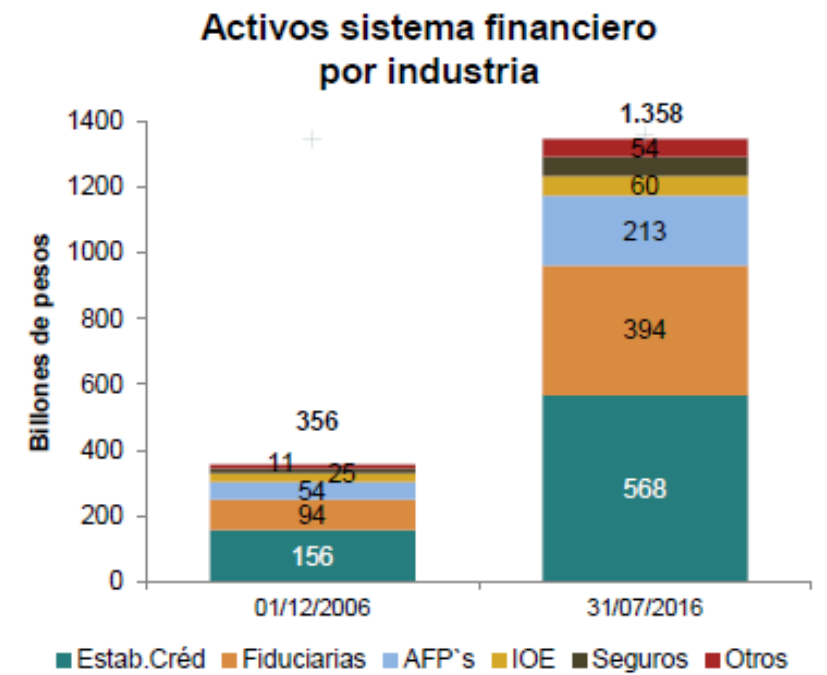

\section{Grafico 2 Activos sistema financiero por industria}

Fuente: Superintendencia Financiera de Colombia

Se evidencia que los establecientes de crédito representan actualmente cerca del $42 \%$ del total de los activos del sistema financiero colombiano, por esto es de vital importancia contar con unos estándares de calidad y control en la gestión de estas instituciones.

\section{Implementación de los anteriores acuerdos de Basilea en Colombia}

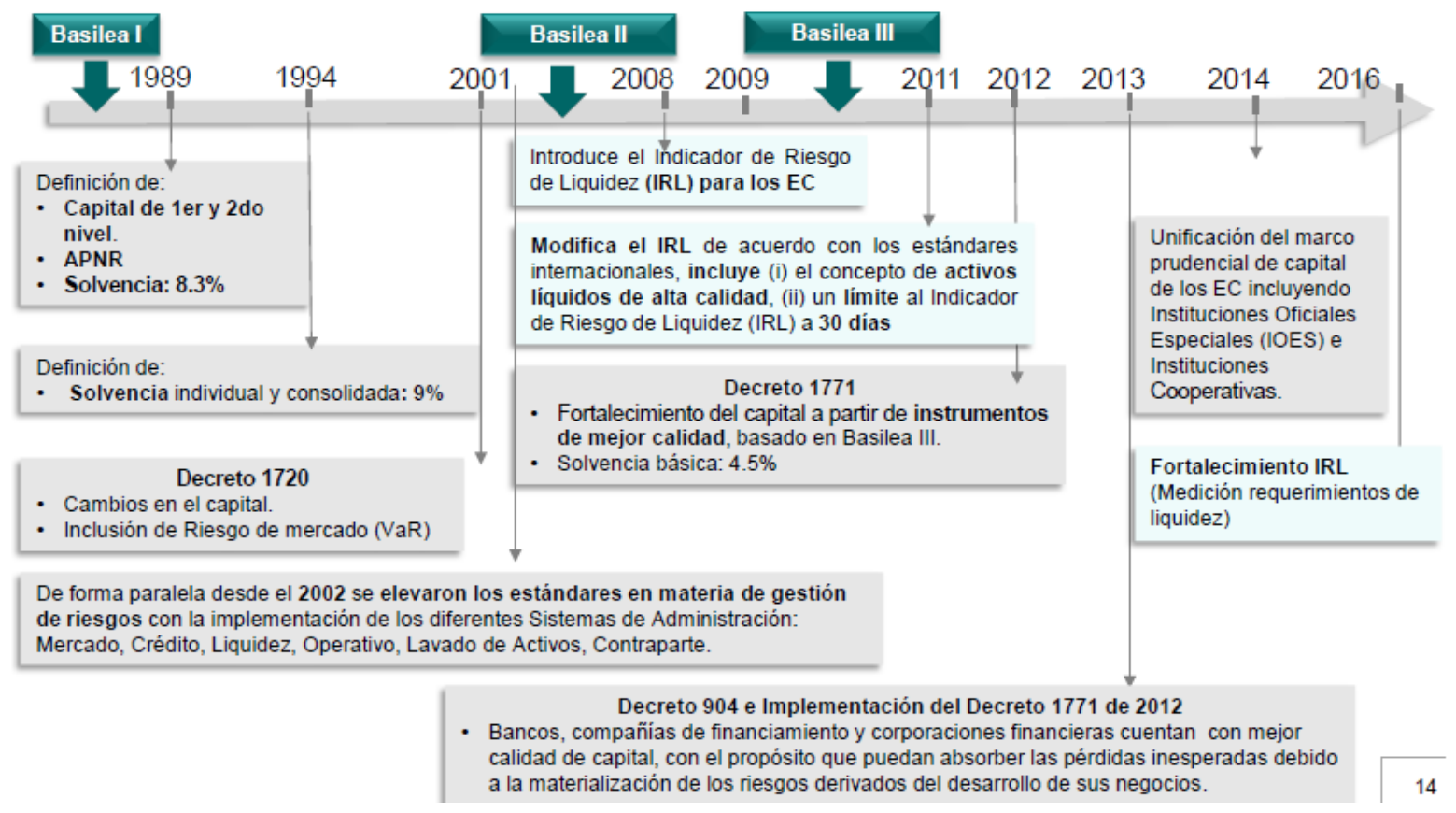

Imagen 2 Línea de tiempo de los Acuerdos de Basilea

Fuente: Superintendencia Financiera de Colombia 
El proceso de implementación y ajustes en Colombia, al igual que en otros países de la región ha sido gradual y ordenado. A continuación, se muestra una línea el tiempo de la implementación de los acuerdos de Basilea:

En el año 1989 (Basilea I) se define los requerimientos de capital del primer y segundo nivel, se definen los activos ponderados por nivel de riesgo y se establece un mínimo de solvencia del $8.3 \%$.

En el año 1994 se introduce el concepto de solvencia individual y consolidad, en 2001 con el decreto 1720 se realizaron cambios en el capital y se prestó gran atención a la medición de riesgo de mercado por medio del VaR. Así mismo en el año 2002 se elevaron los estándares de mediciones de riesgos con la implementación de sistemas de administración que contemplaban el riesgo de crédito (SARC), riesgo de mercado (SARM), riesgo de liquidez (SARL), Riesgo operativo y de contraparte (SARiC), Lavado de activos (SARLAFT).

En el año 2004 se modifican los acuerdos iniciales dando origen a Basilea II, se 2008 se introduce el indicador IRL como medida de riesgo de liquidez para los establecimientos de crédito.

Para el año 2011 ya con una nueva reglamentación vigente (Basilea III) se realizan las modificaciones al indicador de riesgo de crédito IRL de acuerdo con los estándares internacionales, se incluye el concepto de activos líquidos de alta calidad, y se establece un límite al IRL (30 Días).

En el año 20 el gobierno de Colombia publicó el decreto 1771 el cual modifica las reglas con las que se estará calculando la relación de solvencia de los establecimientos de crédito, a su vez busca el fortalecimiento del capital a partir de instrumentos de mejor calidad.

En el año 2013 con el decreto 904 y con la aplicación del decreto 1771 de 2012 se estableció que los participantes del sistema financiero colombiano deben contar con un capital de calidad, con el propósito de que estos puedan absorber perdidas inesperadas debido a la materialización de los riesgos derivados de su negocio. En el año 2016 se realizó una última modificación al indicador de liquidez en busca de su fortalecimiento.

\section{Basilea III En Colombia}

Como se vio anteriormente, Colombia se encuentra en una transición entre los acuerdos de Basilea II y Basilea III a continuación, se va a realizar una descripción del estado de cada uno de los pilares de Basilea en el sistema financiero colombiano.

\section{Pilar I de Basilea "Requerimientos mínimos de capital"}

En cuanto a la calidad del capital, actualmente se adoptaron tres categorías de capital, y se están manejando dos razones de solvencia. Por otro lado, para mantener los niveles de liquidez se introdujo el indicador de riesgo de liquides (IRL), con los siguientes parámetros: 
activos líquidos, activos líquidos de alta calidad y se estableció un límite del 100\% a 30 días. (Superintendencia Financiera de Colombia, 2016).

La Superintendencia Financiera de Colombia en conjunto con el sistema financiero colombino está trabajando para mejorar la calidad del capital creando colchones de capital que puedan hacer frete a posible crisis futuras o posibles eventualidades que requieran liquidez inmediata.

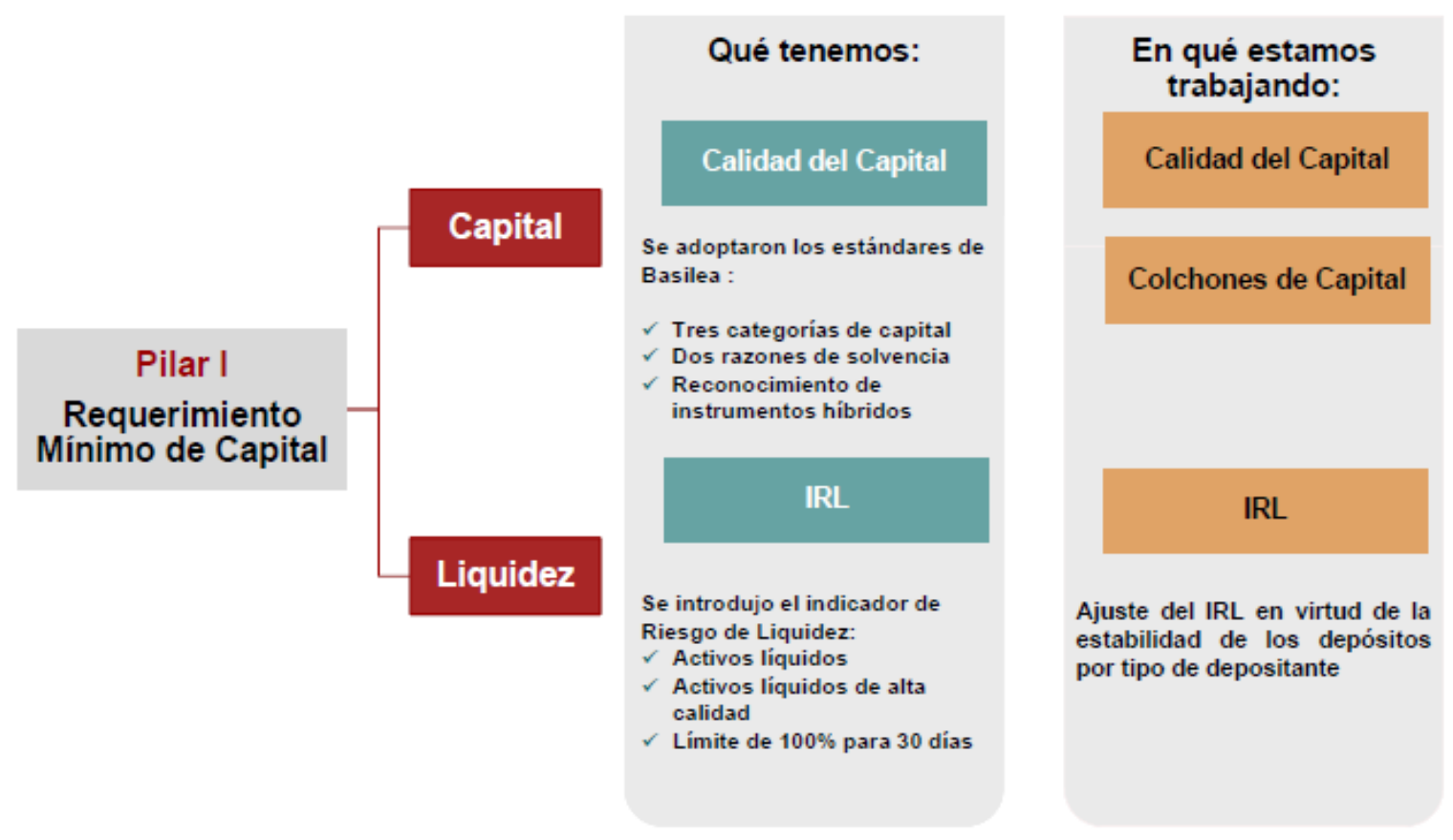

Imagen 3 Requerimiento Mínimo de Capital

Fuente: Superintendencia Financiera de Colombia

Pilar II "Proceso de revisión de Supervisor": en cuanto a este pilar de Basilea, para garantizar el proceso de evaluación de suficiencia del capital, se le dio a la superintendencia financiera la facultad para requerir capital adicional por riegos, esto establecido en el decreto 2392 de 2015. Se estableció un esquema de pruebas de resistencia como un instrumento de gestión del riesgo (Superintendencia Financiera de Colombia, 2015), estas pruebas buscan que las entidades implementen metodologías para identificar y evaluar las vulnerabilidades en escenarios adversos pero probables, como también que adopten planes de acción para su mitigación.

Para realizar una correcta evaluación del supervisor, se implementó el marco integral de supervisión(MIS) Este marco de supervisión describe los principios y conceptos misionales utilizados por la Superintendencia Financiera de Colombia (SFC) para guiar la supervisión que realiza sobre todas las entidades vigiladas. (Superintendencia Financiera de Colombia, 2015) 


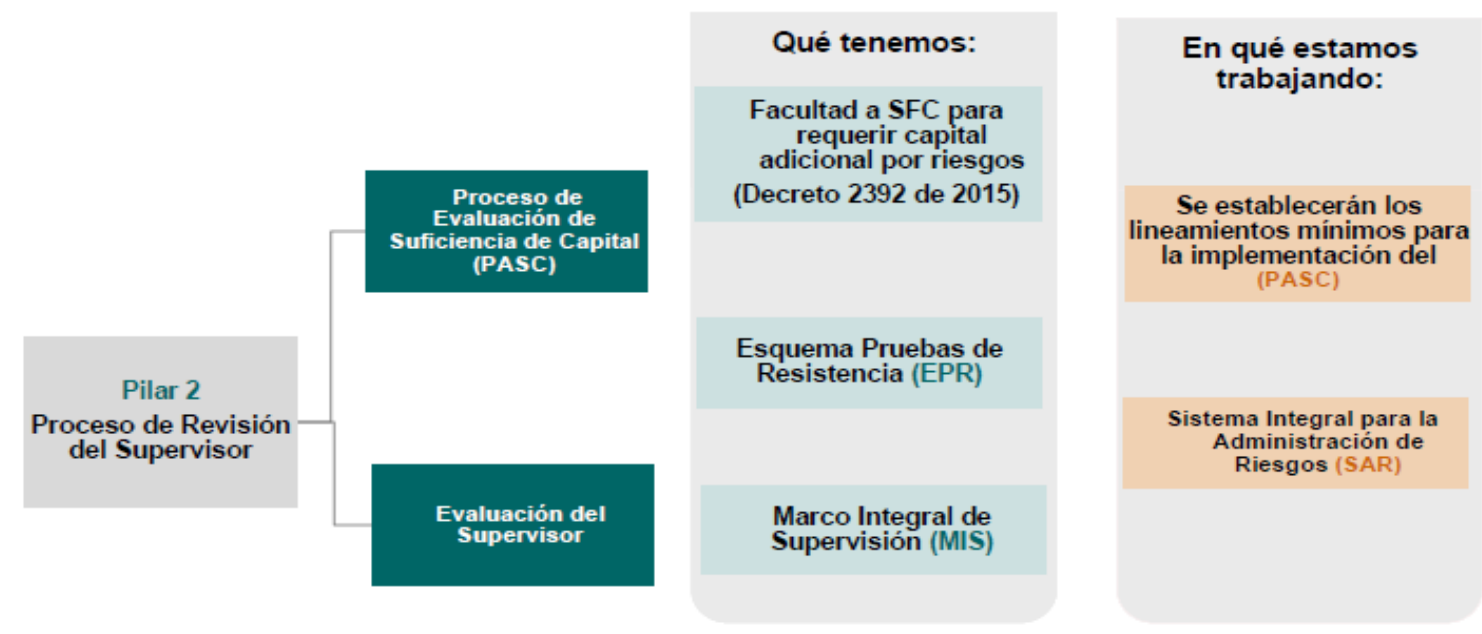

Imagen 4 Proceso de revisión del supervisor

Fuente: Superintendencia Financiera de Colombia

\section{Impacto de la aplicación de los acuerdos de Basilea en el sistema financiero colombiano}

Para tener una noción del impacto que generaría la aplicación de Basilea III en el sistema financiero colombiano, se realizó una revisión documental acerca de simulaciones que se hayan realizado sobre el tema propuesto.

Las simulaciones se basan principalmente en realiza un análisis de sensibilidad de la relación de solvencia ante diferentes escenarios, la relación de solvencia puede entenderse como la capacidad patrimonial de los bancos para respaldar sus activos dado el apalancamiento que tienen con los depósitos del público, es decir es un indicador que cuantifica la cantidad de recursos propios que aportan las entidades financieras en la actividades de intermediación entre ahorradores e inversores (SFC S. f., 2014). En Colombia en el año 2012 se publicó el decreto 1771, en el cual se modifican las reglas actuales con las que se estaba calculando la relación de solvencia de los establecimientos de crédito (Alcaldía de Bogotá, 2012)

Esto se implementa con el principio de contar con un patrimonio adecuado, de calidad y con el potencial de enfrentar tensiones financieras, para así garantizar el buen funcionamiento de las entidades y salvaguardar los depósitos del público. Clavijo, Vera, Malagón y González (2012) explican que este nuevo marco se divide en tres partes el capital regulatorio (Patrimonio Técnico), el cual debe cumplir con los siguientes requisitos:

- Patrimonio Básico Ordinario (PBO) neto de deducciones. Éste comprende los instrumentos de capital de alta disponibilidad. Ellos deben poder utilizarse para enfrentar posibles deterioros en la posición financiera de las entidades frente a choques adversos. Dentro de este capital tangible (Tier 1 core) sobresalen las reservas legales (utilidades retenidas) y las acciones ordinarias. 
- Patrimonio Básico Adicional (PBA). Aquí se incluyen las acciones preferenciales. Así, la suma del PBO neto de deducciones y el PBA equivalen al nivel I (Tier I).

- Patrimonio Adicional (PA). Éste contempla distintos tipos de instrumentos, de menor convertibilidad, pero igualmente válidos como capital. Entre éstos se destacan los bonos subordinados, la valorización de inversiones y las provisiones generales (que se pueden incluir hasta por el $1.25 \%$ de los APR), entre otros. Este componente equivale al nivel II (Tier II).

Estas nuevas definiciones tienen como fin asegurar unos niveles mínimos de patrimonio, de la mejor calidad que les permitan a los establecimientos cubrir las posibles pérdidas derivadas del deterioro de sus activos y respaldar los recursos del público que administran.

Esto se traduce en que se debe realizar una depuración del capital regulatorio, buscando que el patrimonio este compuesto por instrumentos que cumplan con criterios exigentes de permanencia, liquidez y posible absorción de pérdidas. Por tal motivo, la nueva regulación excluye conceptos tales como la valoración de activos fijos, el godwill y los impuestos diferidos netos entre otros, los cuales elevan artificialmente el patrimonio y pueden generar distorsiones en la ecuación contable (Clavijo, Vera, Malagón, \& González, 2012). Asobancaria (2012) estimó que la limpieza del capital bancario en Colombia implicaría una caída en la solvencia por un valor equivalente al 0.85\% del PIB (Casi \$5.3 Billones) respecto del valor observado a diciembre de 2011.

A su vez la asociación nacional de instituciones financieras ANIF, realizó un análisis de sensibilidad del índice de solvencia ante diferente escenario, presentado los resultados en su informe "Basilea iii y el sistema bancario de Colombia: simulaciones sobre sus efectos regulatorios", allí concluyó que ajustes simultáneos e inmediatos en los conceptos de godwill, pasivo pensional, y deuda subordinada resultarían algo extremos para Colombia, pues son bastante exigentes y de hecho lo llevaría en su conjunto por debajo del $9 \%$ de solvencia mínima actual (Clavijo, Vera, Malagón, \& González, 2012). En este sentido, es evidente que la mejor opción sea la implementación gradual de los acuerdos.

Siguiendo con el orden el artículo se realizará la ampliación de la información relacionada con los acuerdos de Basilea en la economía chilena.

Contextualización del sistema financiero chileno.

El sistema financiero chileno está compuesto por fondos de inversión, fondos mutuos, compañías de seguro, fondos de pensiones y entidades bancarias, en la siguiente grafica se muestra la composición en términos de activos del sistema financiero chileno, se evidencia que hay una gran participación de las entidades bancarias dentro del mismo, por ende, se por esto es de vital importancia contar con unos estándares de calidad y control en la gestión de estas instituciones. (hacienda, 2016) 


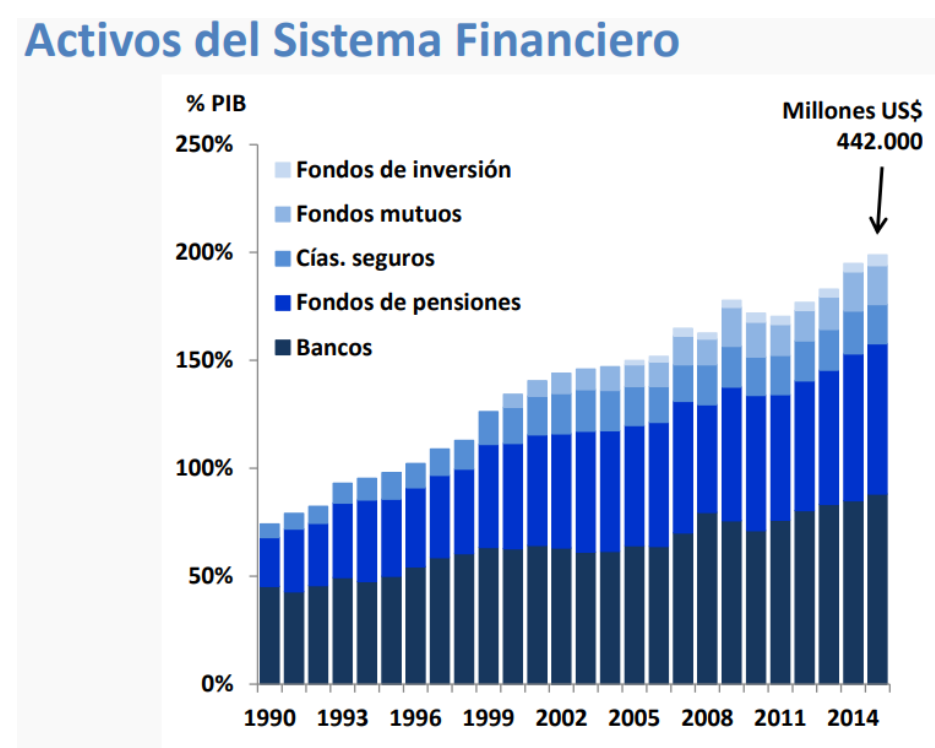

Grafico 3 Activos del sistema financiero

Fuente: (hacienda, 2016)

Según la Superintendencia de Bancos e Instituciones Financieras (SBIF), El sistema financiero chileno está compuesto en la actualidad por 21 bancos establecidos, de dichos bancos hay 14 que se consideran como "Bancos Establecidos en Chile", y 6 Sucursales de Bancos Extranjeros, Finalmente, existe un Banco Estatal, que corresponde al Banco del Estado de Chile . A todos ellos se agrega el Banco Central de Chile que no es fiscalizado por esta Superintendencia.

Implementación de los anteriores acuerdos de Basilea en Chile

En 1988 cuando comienza la normativa de Basilea I, Chile dejó de estar completamente alineado con los estándares internacionales definidos en los acuerdos, aplicando una implementación parcial de Basilea I. Las regulaciones posteriores vinieron a ampliar la brecha entre el país y los principios del Comité de Basilea. (Santander, 2014).

En la transición a Basilea II se presentaron los siguientes resultados que se detallaran por cada uno de los pilares:

- Pilar I: "Requerimientos de capital" El capital mínimo que requiere un banco debe superar el $8 \%$ de los activos ponderados por riesgo, más cargos de capital por concepto de riesgo de mercado y riesgo operacional.

- Pilar II: "Examen del supervisor" En Chile no se cumple este pilar, actualmente la Clasificación de Gestión y Solvencia que realiza regularmente la Superintendencia reúne varios principios pertinentes.

Pilar III: "Disciplina del mercado" Parte de estos requerimientos fueron cubiertos con la adopción de IFRS el año 2008, aún se requieren mayores y mejores reportes por parte del Supervisor. Otro esfuerzo ha sido la normativa sobre gobiernos corporativos. (Santander, 2014). 
$\underline{Y+\} B a s i l e a ~ I I I ~ E n ~ C h i l e ~}$

Los bancos más grandes de Chile están bien posicionados para cumplir con las nuevas reglas de liquidez y financiamiento estable establecidas en Basilea III, dadas sus sólidas franquicias de depósito

y la capacidad de adaptar sus balances, según Fitch Ratings (2016). En cambio, los bancos con una base minorista más pequeña y una mayor dependencia de la financiación mayorista pueden encontrar más difícil cumplir con los requisitos, y sus ganancias podrían sufrir como resultado. (Bnamericas, 2017).

Aunque La adopción de Basilea II y III no ha sido posible en su totalidad por diversas razones, en especial por la necesidad de cambios en la regulación de la ley general de Bancos LGB (ya que todo cambio a las relaciones entre activos y patrimonio implica una modificación a la Ley general de Bancos, y por lo tanto debe pasar por el Congreso), las autoridades aseguran que es el momento para centrar sus esfuerzos en alinearse con la reglamentación vigente de Basilea. Sin embargo, resaltan que estos cambios no serán inmediatos, más allá del trámite legislativo deben pasar varios años para ir avanzando de manera ordenada y sin sobresaltos. (Santander, 2014).

Para lograr esto, el gobierno chileno ha presentado un proyecto de ley que reforma la Ley General de Bancos (LGB) en Chile, este proyecto busca actualizar el actual ordenamiento normativo a los cambios regulatorios consensuados en el ámbito internacional, con el propósito de fortalecer la estabilidad del sistema financiero local. (SBIF, 2018).

La superintendencia de bancos y entidades financieras presenta las siguientes modificaciones a la Ley General de Bancos (SBIF, 2018):

- En términos de la composición de capital, se fija un mínimo de $8 \%$ para el capital regulatorio, el cual se compone de capital básico, capital adicional de nivel 1 y capital de nivel 2.

- En cuanto a la calidad del capital, al capital regulatorio se le deben sumar una serie de buffers o colchones de capital adicional, diseñados para enfrentar riesgos específicos de la institución bancaria o contingencias macroeconómicas. Se establecen los siguientes buffers; Un buffer general y permanente llamado colchón de conservación, este es un requerimiento de capital básico adicional equivalente a $2,5 \%$ de los APR, por lo que en condiciones normales todos los bancos debieran mantener un capital regulatorio mínimo de 10,5\% de los APR. Otro buffer general, pero no permanente, es el colchón contracíclico, que puede variar en el rango de $0 \%$ y 2,5\% de los APR, constituido también sólo con CET1.

- La clasificación de solvencia se redefine en función del cumplimiento efectivo de los distintos requerimientos de capital, de tal forma que el nivel A para bancos que 
cumplen con todas las exigencias incluidos los colchones generales de capital, nivel B para bancos que no cumplen con dichos colchones (por lo que la repartición de dividendos queda total o parcialmente suspendida) pero si con sus requerimientos específicos y $\mathrm{C}$ cuando la posición de solvencia del banco está debilitada y no cumple con el mínimo requerido por la ley, por lo que la administración del banco y el supervisor deberán tomar medidas correctivas tempranas para resolver dicha situación.

- En cuanto al coeficiente de apalancamiento, se establece un $3 \%$, este actúa como respaldo de las medidas de capital basadas en riesgo y proporciona una mayor protección frente al riesgo de modelos y errores de medición. Este requisito puede aumentar hasta $5 \%$ en el caso de bancos de importancia sistémica.

- En cuanto al pilar 2 sobre el proceso de supervisión se establece que, aunque Chile cuenta con un marco de supervisión y regulación financiera robusto, es recomendable fortalecer la independencia y protección legal de las autoridades regulatorias

- Por último, se abarca tercer pilar de Basilea III Disciplina de Mercado, en donde se mantienen las facultades otorgadas a la Superintendencia para requerir información directamente de los bancos o instruir su divulgación al mercado.

Impacto de la aplicación de los acuerdos de Basilea en el sistema financiero chileno En cuanto la implementación de Basilea III, la banca debe modificar, en un plazo hasta septiembre de 2019, el diseño y arquitectura actual de los sistemas de información institucional. Esto conllevará a aumentar la capacidad de almacenamiento y procesamiento de datos y a ajustar los procedimientos de supervisión de la aprobación y monitoreo de metodologías internas desarrolladas por los bancos. (Chile, s.f.).

En junio de 2017, S\&P se refiere en su artículo "Nueva Ley Bancaria en Chile, un paso significativo para impulsar la resiliencia del sistema financiero" a la implementación de Basilea III a través de cambios en la ley, donde menciona que el retraso en la implementación del acuerdo lo ha puesto en el grupo 3, uno de los grupos más elevado de riesgo ${ }^{1}$, siendo el grupo 1 el de mayor riesgo, y grupo 10 el de menor riesgo, lo anterior promoviendo ser el país con mayor riesgo de Latinoamérica. Sin embargo, al haber implementado en otros países de Latinoamérica este acuerdo, facilita el trabajo del regulador chileno reduciendo posibles errores que se puedan presentar durante la implementación.

Ventajas competitivas y retos que afrontan Colombia y Chile con la aplicación de los acuerdos de Basilea.

Una de las principales ventajas competitivas que surge de la aplicación de los acuerdos de Basilea es el fortalecimiento de la solvencia de la banca, mejorando así la competitividad de las entidades financieras tanto a nivel local como internacional, facilitando el acceso a nuevas fuentes de financiamiento, más diversificadas, de menor costo o mayor plazo, contribuyendo

\footnotetext{
${ }^{1}$ Segmentación que evalúa el riesgo de inversión, se califica de 1 a 10, siendo 1 el riesgo más alto y 10 el más bajo
} 
así a una mayor estabilidad del sistema, de esta forma la banca dispondrá de recursos que resulten suficientes para enfrentar en forma más sólida las contingencias e incertezas de la economía global.

Una segunda ventaja que vale la pena resaltar es la armonización en los requerimientos entre filiales de bancos extranjeros y locales, lo que permite eliminar asimetrías regulatorias en la jurisdicción a esto hay que adiciónale que las reformas de Basilea III servirán de plataforma para seguir mejorando la gestión de riesgos, la divulgación de información y las practicas supervisoras.

El director del banco central de pagos, Jaime Caruana (2010) establece que con el pilar II de Basilea se espera mejorar la gestión de riesgos de las entidades, captar el riesgo de las exposiciones fuera de balance y las actividades de totalización; fortalecer los procesos de valoración de los instrumentos financieros, así como diseñar e implementar programas adecuados para la realización de pruebas de resistencia y alinear incentivos para gestionar mejor los riesgos y rentabilidades a largo plazo, incluidas las prácticas remunerativas.

Así mismo resalta que, con el III Pilar de Basilea las entidades regulatorias exigen a los bancos a divulgar todos los componentes de su base de capital regulatorio, las deducciones que aplican y la conciliación completa de las cuentas financieras esto contribuirá a la disciplina de mercado.

En cuanto a las desventajas o retos a los que están expuestos los países, el secretario de la Asociación de Supervisores Bancarios de las Américas, Rudy Araujo, opina que implementar a los estándares de Basilea al pie de la letra añadiría un costo innecesario a los clientes del sector y por ende retrasaría las metas de inclusión financiera. (Dinero, 2017).

Otro de los retos que se enfrenta los acuerdos de Basilea es el constante cambio en los mismos, lo cual no es beneficioso para el sistema financiero pues este necesita estabilidad, adicional a esto nos encontramos en un mundo donde la transformación digital está obligando a transformar los negocios bancarios de forma acelerada y por eso es necesario cerrar rápido el capítulo de los acuerdos de Basilea y enfocarse en la regulación que debe venir con el desarrollo tecnológico.

La implementación de los acuerdos traerá consigo importantes costos que deberán ser soportados por las instituciones financieras a fin de ajustarse a los estándares de capital regulatorio que esta normativa recomienda, esto podría incrementar el costo del financiamiento y fortalecer a las entidades crediticias no financieras, con menor regulación que puedan desestabilizar lo conseguido con los acuerdos. (García, 2017). 


\section{Conclusiones}

Aunque es claro que los acuerdos de Basilea traerán consigo mayor estabilidad y confianza, así como un sistema financiero más robusto y capaz de enfrentar situaciones de estrés, se debe tener en cuenta las generalidades que afecta al sistema financiero de cada economía, y debe aplicarse con la gradualidad necesaria para que el sistema bancario y financiero local pueda asumir el desafío de hacer frente a este nuevo marco regulatorio sin comprometer ni resentir su estabilidad y solvencia. A lo largo del documento se resaltó la importancia de los establecimientos bancarios dentro del sistema financiero tanto en la economía colombiana y chilena, participando en más de un $50 \%$ de los activos del sistema financiero, por esto es de vital importancia contar un sector bancario sólido, regulado y estable. Enfrentar las consecuencias de implementar un nuevo marco regulatorio, es una situación que no es ajena a las economías estudiadas en este documento, Colombia y Chile están en una transición entre Basilea II y Basilea III (Basilea 2.5). Para el caso de la banca colombiana se ha venido haciendo un trabajo mancomunado con el ente regulador, destacándose en aspectos como el nivel de solvencia requerido que, incluso, se sitúa por encima del mínimo regulatorio (9\%) o la inclusión de los instrumentos híbridos como parte del patrimonio técnico.

Con respecto a la economía chilena, los acuerdos de Basilea se han visto retrasados en su aplicación debido a la necesidad de realizar cambios en la regulación de la ley general de Bancos, LGB, (ya que todo cambio a las relaciones entre activos y patrimonio implica una modificación a la Ley general de Bancos, y por lo tanto debe pasar por el Congreso). La economía chilena presentara un proyecto de ley acorde con todos los conceptos básicos de Basilea III, exigiendo más y mejor capital y manteniendo las disposiciones prudenciales adicionales vigentes en la ley actual.

Esto muestra el gran compromiso que tienen las economías estudiadas con la implementación de los acuerdos de Basilea III, pero se debe prestar especial atención a los efectos que estos tendrían en el desarrollo de las actividades económicas de los países en cuestión y que adoptarlas de una forma total e instantánea afectaría tanto a las instituciones financieros como a los consumidores. Por esto se debe adaptar los acuerdos a las necesidades específicas de cada país en vez de adoptarlos al pie de la letra realizando este proceso de forma gradual y controlada. 


\section{Referencias}

Alcaldía de Bogotá. (23 de Agosto de 2012). DECRETO 1771 DE 2012. Obtenido de http://www.alcaldiabogota.gov.co/sisjur/normas/Norma1.jsp?i=48994

Asobancaria. (2010). Basilea III y los impactos preliminares en México. . Obtenido de http://www.asobancaria.com/portal/pls/portal/docs/1/962048.PDF.

Asobancaria. (2012). Una nueva relación de solvencia para la banca colombiana”,. Semana Económica Edición No. 867, Septiembre.

Asociación Nacional de Instituciones Financieras, ANIF. (Noviembre de 2012). BASILEA III Y EL SISTEMA BANCARIO DE COLOMBIA :SIMULACIONES SOBRE SUS EFECTOS REGULATORIOS. Obtenido de http://anif.co/sites/default/files/investigaciones/anifbasilea1112_1.pdf

Banco de Pagos Internacionales. (Julio de 2004). Aplicación de Basilea II: Aspectos prácticos. Obtenido de https://www.bis.org/publ/bcbs109esp.pdf

BIS. (s.f.). Obtenido de https://www.bis.org/bcbs/basel3/b3_bank_sup_reforms_es.pdf

Bnamericas. (24 de Noviembre de 2017). Grandes bancos de Chile listos para Basilea III. Obtenido de https://www.bnamericas.com/es/noticias/banca/grandes-bancos-de-chile-listos-parabasilea-iii

Caruana, J. (19 de noviembre de 2010). La importancia de Basilea III para los mercados financieros. Obtenido de https://www.bis.org/speeches/sp101125_es.pdf

CEPAL. (2016). Panorama de la Inserción Internacional. Obtenido de https://repositorio.cepal.org/bitstream/handle/11362/40744/1/S1601274_es.pdf

Chile, S. d. (s.f.). Obtenido de https://www.sbif.cl/sbifweb3/internet/archivos/publicacion_12021.pdf

Clavijo, S., Vera, A., Malagón, D., \& González, A. (2012). Basilea III y el Sistema Bancario de Colombia: Simulaciones sobre sus efectos regulatorios. ANIF.

Dinero. (2017). el problema de implementar a 'rajatabla' los estándares de Basilea le añadiría un costo innecesario a los clientes del sector y por ende retrasaría las metas de inclusión financiera. Revista Dinero, 2.

Economipedia. (s.f.). Obtenido de http://economipedia.com/definiciones/acuerdos-de-basilea.html

El Espectador. (17 de Septiembre de 2008). Colombia y la crisis financiera internacional. El espectador.

García, E. (2017). Los desafíos y riesgos de la incorporación de las normas de Basilea III a la nueva Ley General de Bancos. El mercurio, 1.

hacienda, M. d. (04 de mayo de 2016). Comisión Especial Investigadora de la actuación de los organismos públicos competentes en relación al eventual fraude de empresas de 
inversiones. Obtenido de

https://www.camara.cl/pdf.aspx?prmID=52942\&prmTIPO=DOCUMENTOCOMISION

Heresi, R., \& Ffrench-Davis, R. (2011). La economía chilena frente a la crisis financiera: respuestas contra-cíclicas y desafíos pendientes. Obtenido de Facultad de Economía y Negocios Universidad de Chile:

http://www.econ.uchile.cl/uploads/publicacion/001e3a0544728fa124a18600759e7881a1dce 8cf.pdf

Jiménez, C., Held, G., \& Romero, L. (07 de 2008). Capital regulatorio de los bancos en la implementación de Basilea II. Santiago: Superintendencia de Bancos e Insituciones Financieras . Obtenido de https://www.sbif.cl/sbifweb/internet/archivos/publicacion_7006.pdf

Mandi, C. (23 de 02 de 2012). Edital de Basileia 3 trouxe novidades positivas, avalia. Obtenido de http://www.valor.com.br/financas/2539332/edital-de-basileia-3-trouxe-novidades-positivasavalia-febraban\#ixzz273AQLHs5

Powerdata. (s.f.). blog.powerdata. Obtenido de https://blog.powerdata.es/el-valor-de-la-gestion-dedatos/bid/307125/qu-son-los-acuerdos-de-basilea-basilea-i-basilea-ii-y-basilea-iii

Santander, B. (Enero de 2014). Basilea III: ¿Qué es y por qué es necesario adoptarla? Obtenido de https://saladecomunicacion.santander.cl/wpcontent/uploads/2016/03/1393441904140204_PolicyPaper6_BasileaIII.pdf

SBIF, S. d. (2018). Implementación de Basilea III en Chile: Fundamentos y Desafíos.

SFC, S. f. (2014). Patromonio tecnico y niveles minimos de solvencia Capitulo 13. Obtenido de https://www.superfinanciera.gov.co/descargas?...13patrimoniotecnicoreminsolvencia

SFC, S. F. (Octubre de 2016). AVANCES EN SUPERVISIÓN Y REGULACIÓN DEL SISTEMA FINANCIERO COLOMBIANO. Obtenido de https://m.superfinanciera.gov.co/descargas?com=institucional\&name...pdf

Sotelo, R. J. (10 de 06 de 2008). Economía y Finanzas para Todos . Obtenido de http://blog.pucp.edu.pe/blog/renzojimenez/2008/06/10/el-comite-de-basilea-y-susprincipios-basicos/

Sotelsek Salem, D., \& Pavón Cuéllar, L. (2012). Evolución de los Acuerdos de Basilea: diagnóstico de los estándares de regulación bancaria internacional. Economía UNAM, 29-50. Obtenido de http://www.economia.unam.mx/publicaciones/nueva/econunam/25/03sotelsekypavon.pdf

Superintendecia de Banca, Seguros y AFP . (s.f.). Basilea II: El Nuevo Acuerdo de capital. Obtenido de http://www.sbs.gob.pe/Portals/0/jer/REGUL_PROYIMP_BASIL_FUNSBS/BasileaIIIntroduccion-JPoggi-MLuy.pdf

Superintendencia Financiera de Colombia. (2015). Marco Integral de Supervisión. Obtenido de https://m.superfinanciera.gov.co/descargas?com...marcointegralsupervision.docx 
Superintendencia Financiera de Colombia. (17 de Noviembre de 2016). XV Congreso de Riesgo Financiero: Una Mirada Global.

Superintendencia Financiera de Colombia, S. (20 de Agosto de 2015). Pruebas de resistencia como instrumento para la gestion de riesgos.

Trujillo, J. (Julio de 2015). Acuerdos de Basilea III. Un Nuevo Marco Global en Términos de Liquidez. Obtenido de https://repositorio.comillas.edu/xmlui/bitstream/handle/11531/6425/TFM000217.pdf?seque nce $=1$

Uribe, J. D. (Enero de 2013). Revista del Banco de la República. Obtenido de http://www.banrep.gov.co/sites/default/files/publicaciones/archivos/rbr_nota_1023.pdf 